\title{
DECOMPOSING THE COMPLETE GRAPH INTO HAMILTONIAN PATHS (CYCLES) AND 3-STARS
}

\author{
Hung-ChiH LeE \\ Department of Information Technology \\ Ling Tung University \\ Taichung 40852, Taiwan \\ e-mail: birdy@teamail.ltu.edu.tw \\ AND \\ Zhen-Chun Chen \\ Department of Applied Mathematics \\ National Chiao Tung University \\ Hsinchu 300, Taiwan \\ e-mail: amco0624@yahoo.com.tw
}

\begin{abstract}
Let $H$ be a graph. A decomposition of $H$ is a set of edge-disjoint subgraphs of $H$ whose union is $H$. A Hamiltonian path (respectively, cycle) of $H$ is a path (respectively, cycle) that contains every vertex of $H$ exactly once. A $k$-star, denoted by $S_{k}$, is a star with $k$ edges. In this paper, we give necessary and sufficient conditions for decomposing the complete graph into $\alpha$ copies of Hamiltonian path (cycle) and $\beta$ copies of $S_{3}$.
\end{abstract}

Keywords: decomposition, complete graph, Hamiltonian path, Hamiltonian cycle, star.

2010 Mathematics Subject Classification: 05C70, 05C38.

\section{REFERENCES}

[1] A. Abueida, S. Clark and D. Leach, Multidecomposition of the complete graph into graph pairs of order 4 with various leaves, Ars Combin. 93 (2009) 403-407.

[2] A. Abueida and M. Daven, Multidesigns for graph-pairs of order 4 and 5, Graphs Combin. 19 (2003) 433-447.

doi:10.1007/s00373-003-0530-3 
[3] A. Abueida and M. Daven, Multidecompositons of the complete graph, Ars Combin. 72 (2004) 17-22.

[4] A. Abueida and M. Daven, Multidecompositions of several graph products, Graphs Combin. 29 (2013) 315-326.

doi:10.1007/s00373-011-1127-x

[5] A. Abueida, M. Daven and K.J. Roblee, Multidesigns of the $\lambda$-fold complete graph for graph-pairs of order 4 and 5, Australas. J. Combin. 32 (2005) 125-136.

[6] A. Abueida and C. Hampson, Multidecomposition of $K_{n}-F$ into graph-pairs of order 5 where $F$ is a Hamilton cycle or an (almost) 1-factor, Ars Combin. 97 (2010) 399-416.

[7] A. Abueida and C. Lian, On the decompositions of complete graphs into cycles and stars on the same number of edges, Discuss. Math. Graph Theory 34 (2014) $113-125$. doi:10.7151/dmgt.1719

[8] A. Abueida and T. O'Neil, Multidecomposition of $\lambda K_{m}$ into small cycles and claws, Bull. Inst. Combin. Appl. 49 (2007) 32-40.

[9] B. Alspach, The wonderful Walecki construction, Bull. Inst. Combin. Appl. 52 (2008) 7-20.

[10] F. Beggas, M. Haddad and H. Kheddouci, Decomposition of complete multigraphs into stars and cycles, Discuss. Math. Graph Theory 35 (2015) 629-639. doi:10.7151/dmgt.1820

[11] J. Bosák, Decompositions of Graphs (Kluwer, Dordrecht, Netherlands, 1990).

[12] D. Bryant, Packing paths in complete graphs, J. Combin. Theory Ser. B 100 (2010) 206-215. doi:10.1016/j.jctb.2009.08.004

[13] P. Hell and A. Rosa, Graph decompositions, handcuffed prisoners and balanced Pdesigns, Discrete Math. 2 (1972) 229-252. doi:10.1016/0012-365X(72)90005-2

[14] S. Jeevadoss and A. Muthusamy, Decomposition of complete bipartite graphs into paths and cycles, Discrete Math. 331 (2014) 98-108. doi:10.1016/j.disc.2014.05.009

[15] S. Jeevadoss and A. Muthusamy, Decomposition of product graphs into paths and cycles of length four, Graphs Combin. 32 (2016) 199-223. doi:10.1007/s00373-015-1564-z

[16] S. Jeevadoss and A. Muthusamy, Decomposition of complete bipartite multigraphs into paths and cycles having $k$ edges, Discuss. Math. Graph Theory 35 (2015) $715-731$. doi:10.7151/dmgt.1830

[17] R. Laskar and B. Auerbach, On decomposition of r-partite graphs into edge-disjoint Hamilton circuits, Discrete Math. 14 (1976) 265-268. doi:10.1016/0012-365X(76)90039-X 
[18] H.-C. Lee, Multidecompositions of complete bipartite graphs into cycles and stars, Ars Combin. 108 (2013) 355-364.

[19] H.-C. Lee, Decomposition of the complete bipartite multigraph into cycles and stars, Discrete Math. 338 (2015) 1362-1369. doi:10.1016/j.disc.2015.02.019

[20] H.-C. Lee and Z.-C. Chen, Maximum packings and minimum coverings of multigraphs with paths and stars, Taiwanese J. Math. 19 (2015) 1341-1357. doi:10.11650/tjm.19.2015.4456

[21] H.-C. Lee and C. Lin, Balanced star decompositions of regular multigraphs and $\lambda$ fold complete bipartite graphs, Discrete Math. 301 (2005) 195-206. doi:10.1016/j.disc.2005.04.023

[22] H.-C. Lee and J.-J. Lin, Decomposition of the complete bipartite graph with a 1factor removed into cycles and stars, Discrete Math. 313 (2013) 2354-2358. doi:10.1016/j.disc.2013.06.014

[23] J.-J. Lin, Decompositions of multicrowns into cycles and stars, Taiwanese J. Math. 19 (2015) 1261-1270. doi:10.11650/tjm.19.2015.3460

[24] C. Lin, J.-J. Lin and T.-W. Shyu, Isomorphic star decomposition of multicrowns and the power of cycles, Ars Combin. 53 (1999) 249-256.

[25] H.M. Priyadharsini and A. Muthusamy, $\left(G_{m}, H_{m}\right)$-multifactorization of $\lambda K_{m}, \mathrm{~J}$. Combin. Math. Combin. Comput. 69 (2009) 145-150.

[26] H.M. Priyadharsini and A. Muthusamy, $\left(G_{m}, H_{m}\right)$-multidecomposition of $K_{m, m}(\lambda)$, Bull. Inst. Combin. Appl. 66 (2012) 42-48.

[27] T.-W. Shyu, Decomposition of complete graphs into paths and stars, Discrete Math. $310(2010)$ 2164-2169. doi:10.1016/j.disc.2010.04.009

[28] T.-W. Shyu, Decompositions of complete graphs into paths and cycles, Ars Combin. 97 (2010) 257-270.

[29] T.-W. Shyu, Decomposition of complete graphs into paths of length three and triangles, Ars Combin. 107 (2012) 209-224.

[30] T.-W. Shyu, Decomposition of complete graphs into cycles and stars, Graphs Combin. 29 (2013) 301-313. doi:10.1007/s00373-011-1105-3

[31] T.-W. Shyu, Decomposition of complete bipartite graphs into paths and stars with same number of edges, Discrete Math. 313 (2013) 865-871. doi:10.1016/j.disc.2012.12.020

[32] D. Sotteau, Decomposition of $K_{m, n}\left(K_{m, n}^{*}\right)$ into cycles (circuits) of length $2 k$, J. Combin. Theory Ser. B 30 (1981) $75-81$. doi:10.1016/0095-8956(81)90093-9 
[33] M. Tarsi, Decomposition of complete multigraphs into stars, Discrete Math. 26 (1979) 273-278.

doi:10.1016/0012-365X(79)90034-7

Received 12 January 2018

Revised 14 May 2018

Accepted 14 May 2018 